\title{
Enhancing Collaborative Manipulation Through the Use of Feedback and Awareness in CVEs
}

\author{
Arturo S. García, José P. Molina, Diego Martínez and Pascual González \\ Laboratory of User Interaction and Software Engineering (LoUISE). University of Castilla-La Mancha (Spain)
}

\begin{abstract}
In the research community, Collaborative Virtual Environments (CVEs) developers usually refer to the terms awareness and feedback as something necessary to maintain a fluent collaboration when highly interactive task have to be performed. However, it is remarkable that few studies address the effect that including special kind of awareness has on the task performance and the user experience.This paper proposes how to face the implementation of awareness in order to be taken into account early in the development of a CVE. In addition, it is also described an experiment that was carried out to evaluate the effect of providing some visual cues, showing that users tend to make more mistakes when they are not provided.
\end{abstract}

Index Terms - Awareness, collaborative work, feedback, virtual reality.

\section{INTRODUCTION}

Nowadays, Collaborative Virtual Environments (CVEs) are experiencing an increasing interest by both users and media. It is mainly due to its more commercial side, leisure and entertainment, where titles like World of Warcraft or Second Life bring together millions of people around the world. However, the interaction within such systems is very limited, and does not make use of the advantages of direct manipulation that immersive Virtual Reality (VR) provides, since there is little object-focused interaction.

Moreover, building CVEs is not an easy task, the problems of developing VR applications [4] must be added to those that arise when dealing with Computer-Supported Collaborative Work systems (CSCW) [6]. The interaction in CVEs is especially important, since several challenges must be faced when implementing real collaborative interaction. Some of them are:

- Problems with the usability of VR devices, due to the lack of maturity of the technology.

- Technical problems related to the implementation of CVEs and to the consistency maintenance of the simulations connected.

- Perception problems related to the way that important information is presented to the users, such as information concerning the state of the world and its objects, the actions of the other users, etc.

Manuscript Received on 23 June 2008.

E-mail: arturo@dsi.uclm.es
Given these problems, this paper aims to address the problem of perception, assuming that some problems with devices and communication networks will be present, and that they will probably hinder the user experience.

Furthermore, the experiment described in this paper intends to address the study of a more complex and realistic way of interaction than the one that is usually supported in some CVEs, the one that [13] call "closely-coupled collaboration". This kind of interaction was chosen due to the challenges posed to the interaction designer, especially due to the lack of haptic feedback. This context requires the revision of the most popular interaction techniques -virtual hand, ray casting, etc.- and also to pay special attention to feedback and user awareness. It is assumed that the problems will be bigger when greater synchronization is required by the task.

This paper is structured as follows: the next section gives an introduction to the main topics of this research, citing the main problems in the field and the main contributions found in the literature. Section three details the approximation followed to tackle with these problems. Section four describes the first experiment carried out following the approximation previously commented. Finally, the last section sums up this work with its main conclusions and its extension in a future work, giving the basis for a wider experiment.

\section{RELATED WORK: COLLABORATIVE INTERACTION, AWARENESS AND FEEDBACK}

Collaborative interaction has been previously studied by [3] in haptics, and [10], [12], [13] and [16] in interaction, among other authors. These and other authors frequently refer to the terms feedback and awareness as something necessary to maintain a fluent collaboration in CVEs, and also as something useful to avoid fragmentation of the collaborative workflow [15], [16], [18] and [20]. It is also mentioned that adding this feedback late in the development process could lead to redesign the application [9].

Collaborative interaction has been classified by different authors during the short history of CVEs. In 1999, [7] classifies previous work at 3 levels, directly related to the degree of collaboration available to users. In the first level, only a simple communication between users is available, while in the second, individual changes of the shared world are allowed. Finally, 

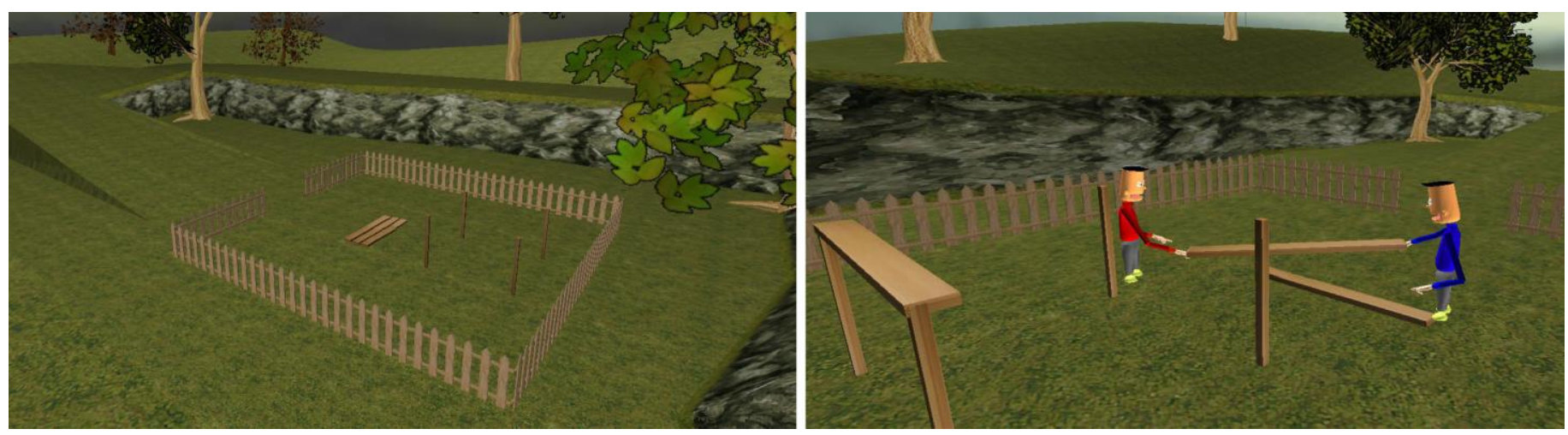

Fig. 1. Virtual environment that replicates the Gazebo of Roberts et al.

TABLE 1: MAPPING BETWEEN OTTO AND MARGERY CLASSIFICATIONS OF COLLABORATIVE INTERACTION AND LEVELS OF COLLABORATION.

\begin{tabular}{|c|c|}
\hline Otto (2006) & Margery (1999) \\
\hline- & Level 1 \\
\hline $\begin{array}{c}\text { Sequential manipulation of } \\
\text { distinct object attributes }\end{array}$ & Level 2 \\
\hline $\begin{array}{c}\text { Sequential manipulation of } \\
\text { the same object attributes }\end{array}$ & Level 2 \\
\hline $\begin{array}{c}\text { Concurrent manipulation of } \\
\text { distinct object attributes }\end{array}$ & Level 3.1 \\
\hline $\begin{array}{c}\text { Concurrent manipulation of } \\
\text { the same object attributes }\end{array}$ & Level 3.2 \\
\hline
\end{tabular}

concurrent changes of different and the same attributes of an object can be performed (levels 3.1 and 3.2).

In 2006, [9] proposed a new classification based on some comments made in [13] and [14]. This classification was only focused on collaborative manipulation, identifying four groups. Despite differences in their approach, a correspondence between the classification of Otto (focusing on collaborative manipulation) and Margery (focusing on levels of collaboration) can be established, as table 1 shows.

Moreover, [2] carried out a study of feedback in VR desktop applications, identifying both types and its basic elements. However, regarding desktop CVEs, few authors have focused their efforts on studying what kind of sensory cues may be needed in such systems, being this number even lower in the case of immersive CVEs.

Given these facts, and given the fact that up to our knowledge there are no work related to what kind of feedback has to be showed, and how it should be done, this paper focuses on how to improve the collaborative interaction using awareness and feedback. It is true that [5] identify some problems and propose some ideas to solve them, but their work is not related to collaborative interaction, just general problems.

At this point, a definition of awareness and its relationship with feedback is given. This is due to the fact that there are different definitions of the same concepts in literature [17]. As awareness, the broadest definition has been taken, the fact of providing the user with enough information to get a vision of what is happening around them. Later in this paper, a classification of awareness will be presented, this time focusing on collaborative interaction. In addition, feedback is understood as the opportunity to guide the user through the use of the application [2].

Moreover, and as we face an environment where several users share objects and perform simultaneous actions on them, the basic elements of feedback identified by Barrilleaux will be used to highlight the actions that all users have to know (they need to be aware of).

\section{OUR APPROACH: HIERARCHICAL TASK ANALYSIS AND AWARENESS CLASSIFICATION}

To cover what in literature is identified as the need to take feedback and awareness into account early in the development process of a CVE, it is proposed to start from the tasks that users must perform in the CVE. These tasks can be divided into subtasks using the HTA (Hierarchical Task Analysis [1]). The aim is to identify the simplest tasks.

Moreover, it is proposed to classify the awareness according to the characteristics of CVEs and focusing on closely-coupled collaboration tasks (table 2). The different groups of awareness identified can be found in the first column. The second one groups the most important events or actions that can be highlighted, while the third column lists some expected benefits of its use. So, this table is intended to help in the selection of the right actions to highlight, having in mind the benefits that this would get.

Once the lowest level tasks have been identified (after the HTA creation), it is possible to study what kind of awareness would be important for each of them (trying not to show too much information to the user, and using it only when it is really needed), considering the recommendations on what actions should be highlighted to improve the user experience. At this point, it is also possible to recommend which basic elements of feedback, from those identified by [2], can be used.

These recommendations can lead a CVE developer who has to include highly interactive tasks in their system to design the interaction in a more usable way. Some of these recommendations were already identified in previous work, but 
TABLE 2: TABLE SUMMARIZING THE PROPOSAL OF AWARENESS NEEDED TO IMPROVE THE USER EXPERIENCE DURING A SIMULATION THAT INVOLVES COLLABORATIVE INTERACTION.

\begin{tabular}{|c|c|c|}
\hline Kinds of awareness & Things to highlight & Expected benefits \\
\hline \multirow{3}{*}{$\begin{array}{l}\text { Object state } \\
\text { awareness }\end{array}$} & Selection (who is selecting) & $\begin{array}{l}\text { Useful for a multiple selection or as a mean of showing what object a user is focused on. } \\
\text { It avoids fragmentation of the collaborative workflow by knowing what object a user is } \\
\text { referring to when speaking or giving instructions. }\end{array}$ \\
\hline & Ownership & $\begin{array}{l}\text { Useful when the owner of an object is important (the concept of "owner" can refer to the } \\
\text { user who created it or the one who is acting on it at the moment). }\end{array}$ \\
\hline & Attribute modification & Useful in teaching situations or in collaborative modifications. \\
\hline \multirow{3}{*}{ Task awareness } & $\begin{array}{l}\text { Participants (users that take } \\
\text { part, field of view) }\end{array}$ & $\begin{array}{l}\text { Users can focus on the task knowing where the participants are and what they are } \\
\text { looking at. It avoids fragmentation of the collaborative workflow. }\end{array}$ \\
\hline & Following action possible & $\begin{array}{l}\text { Avoids fragmentation of the collaborative workflow, just a quick look to the } \\
\text { surroundings would be enough to know what to do next. }\end{array}$ \\
\hline & Task status & $\begin{array}{l}\text { Keep the participants informed about the status of the task, subtasks fulfilled and } \\
\text { subtasks pending, and even risks in the fulfillment of the task. }\end{array}$ \\
\hline \multirow{3}{*}{ World awareness } & Position of important objects & \multirow{2}{*}{ Makes it easy to find them. } \\
\hline & Position of users & \\
\hline & Structure of the world & Makes it easy to locate the user in the virtual environment. \\
\hline \multirow{3}{*}{ Group awareness } & Members on-line & Easy way to know if some user is connected. \\
\hline & Actions of the group members & Makes it easy to know what the group members are doing. \\
\hline & Position of the group members & Makes it easy to find them. \\
\hline \multirow[t]{2}{*}{ Social awareness } & Conversation & $\begin{array}{l}\text { Useful to explain what to do next, in teaching simulations, instruction, to avoid } \\
\text { problems when interacting, etc. }\end{array}$ \\
\hline & Gestures (body language) & Allows a user to get extra information of the intentions, mood, etc. of other users. \\
\hline \multirow{2}{*}{ System awareness } & Network latency & $\begin{array}{l}\text { Users can avoid collaborative interactions when the network latency is high, and take } \\
\text { them up again when the network problems finish. }\end{array}$ \\
\hline & System stability & $\begin{array}{l}\text { This information is useful to solve any inconsistent state of the world, by for example, } \\
\text { repeating some actions. }\end{array}$ \\
\hline
\end{tabular}

sparingly, not focusing on them and not collecting them as here. Moreover, it is not the purpose of this study to replace the existing classifications of awareness in the CSCW field.

\section{FIRST EXPERIMENT}

As a first approximation to the problems of implementing "closely-coupled collaboration", it was decided to conduct an experiment using the task of constructing a Gazebo, as [13] did. The reason for this choice was that the authors have been using it for a long time as a benchmark in their studies and because this task requires a close collaboration between users.

Thus, according to the description of the whole task of building a Gazebo, the authors identified 4 main subtasks: planning and instructing, passing a tool, moving a beam and fixing a beam. Among these 4 subtasks, the one that we considered the more important for the realization of our study was "moving a beam" as it is the only one in which there is a "concurrent sharing of the same attributes".

Therefore, our study consisted of picking a beam up between two users and placing it on other beams that were already located elsewhere in the virtual scene (Fig. 1).

\subsection{Hierarchical Task Analysis and awareness}

Following the recommendations of section 3, a study of the task that was identified as the most important was conducted. The first step was to rename the original task of Roberts "moving a beam" as "placing a heavy beam" because we think that this name describe the task better, and it also avoids misunderstanding with the subtask "Move the beam". The next step was to break the main task down into the subtasks that formed it (Fig. 2).

For this first experiment, we decided to focus our attention on the subtask "Move the beam" because we thought that users would need more help when performing this subtask, since it is the only one that implies close collaboration between both users.

At this point, and thinking about how to support the realization of the task, we had a look at the interaction in the original Gazebo experiment. However, the related papers do not specify how the interaction techniques to manipulate the beam at a time were implemented. Hence, a variation of the Virtual-Hand technique that used an elastic band that binds the user hand to the object was implemented (Fig. 3). Moreover, during a collaborative manipulation, the position of the object will be the middle of the position of the users' hands involved in that manipulation.

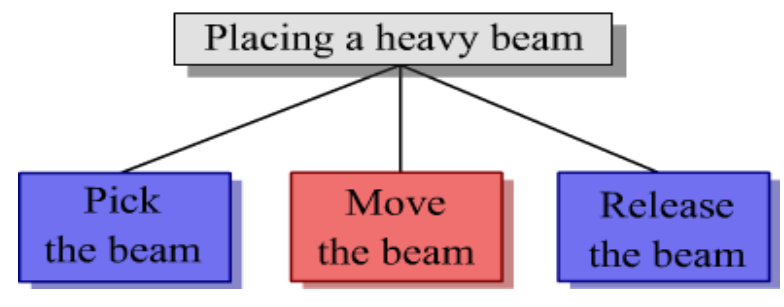

Fig. 2. HTA of the task of moving and placing a heavy beam in the Gazebo experiment. 

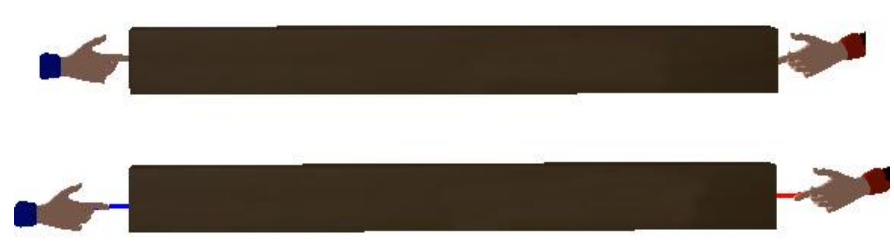

Fig. 3. Variation of the Virtual-Hand interaction technique adapted to collaborative interaction.

In order to make the task more demanding, it was decided that moving the user hand away from the object would make the elastic band break.

The feedback cues introduced tried to improve the performance of the task, so two situations that required additional information were identified: keep the user informed about where their partner is trying to move the beam to (object state awareness, group awareness), and show if the beam is close to fall (task awareness). The first was implemented using an arrow that pointed in the direction of the movement of the user hand, and the second using a visual signal indicating that the beam was close to fall (Fig. 4).

\subsection{CVE system architecture}

This experiment was developed over an architecture implemented from scratch. The most important characteristic is the use of a hybrid network architecture (Fig. 5), where a server redirects all messages exchanged by the clients. However, during a collaborative manipulation, messages related to the negotiation of the final position of the object are only exchanged among participants involved this manipulation (point 1 in Fig. 5). After this, the final position is sent to the server (point 2) and then forwarded to the rest of clients (point 3).

As regards the software platform chosen, the development of the system mostly relied on VRJuggler [19] and OpenSG [8]. Finally, a free and widely used library for network games, Raknet [11], was selected for the transmission of data over the network.
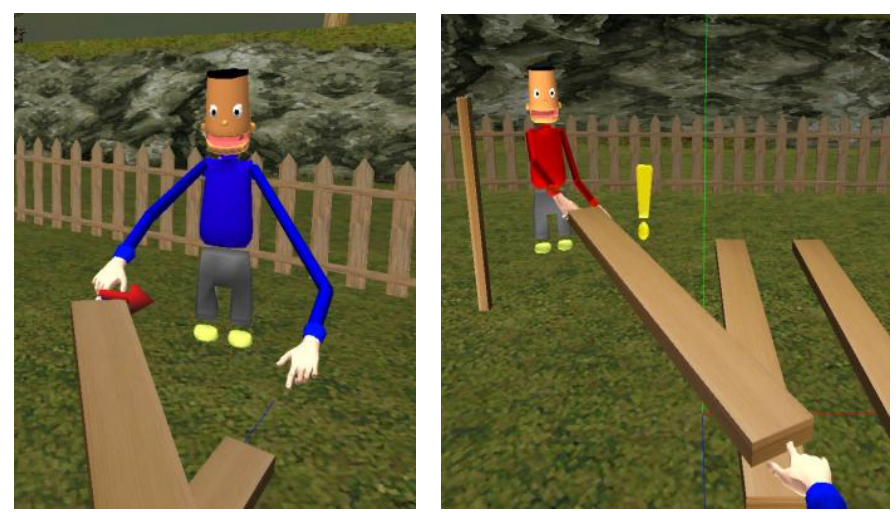

Fig. 4. Feedback cues introduced in the first experiment: movement of the user's partner (left) and visual signal indicating that the beam is close to fall (right).

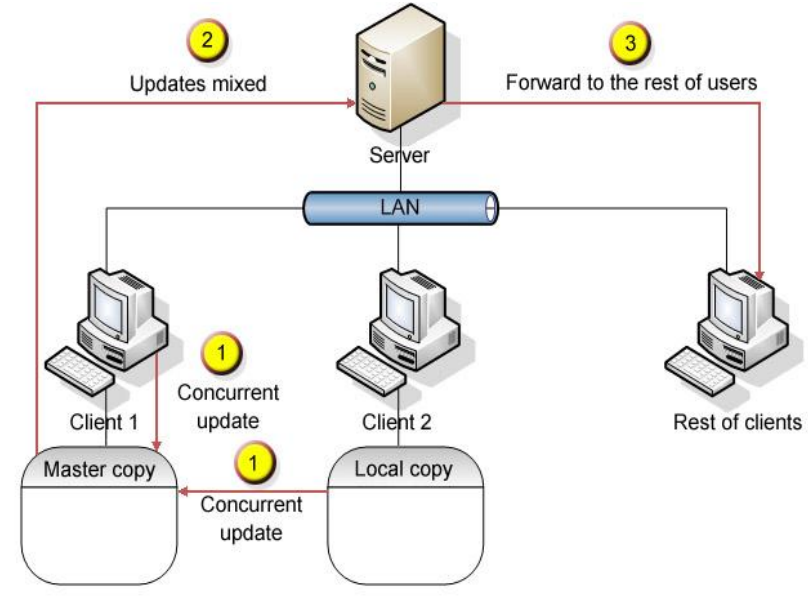

Fig. 5. Network architecture and concurrent updates management.

\subsection{Experiment design}

The experiment was run on a $100 \mathrm{Mbps}$ Ethernet-based local area network connecting three PCs through a hub (two clients and a server). The network was isolated from any other local or wide area network, such as Internet, so no other network traffic was generated apart from the messages that these three PCs sent to each other.

Regarding the VR equipment used, two symmetrical immersive systems were set out because, as Roberts et al., we reckon that "closely-coupled collaboration" is very difficult to achieve in a fluent way using desktop systems. The input and output VR devices used were an Ascension Flock of Birds tracking system with three trackers, a pair of Fakespace Pinch Gloves and a VR1280 stereoscopic head mounted display.

About the participants, the experiment was carried out at PartyQuijote 2008, a LAN party that took place in July 2008 in Albacete, and where over 600 computer enthusiasts met. From them, 40 people, aged from 16 to 52 years, took part in the experiment, in pairs, thus making a total amount of 20 couples (Fig. 6).

Thus, some couples had the previously commented visual cues, and others do not. The hypothesis of this first experiment was that the frequency of beam falls during the performance of the task would be greater in the couples who did not have the visual cues.
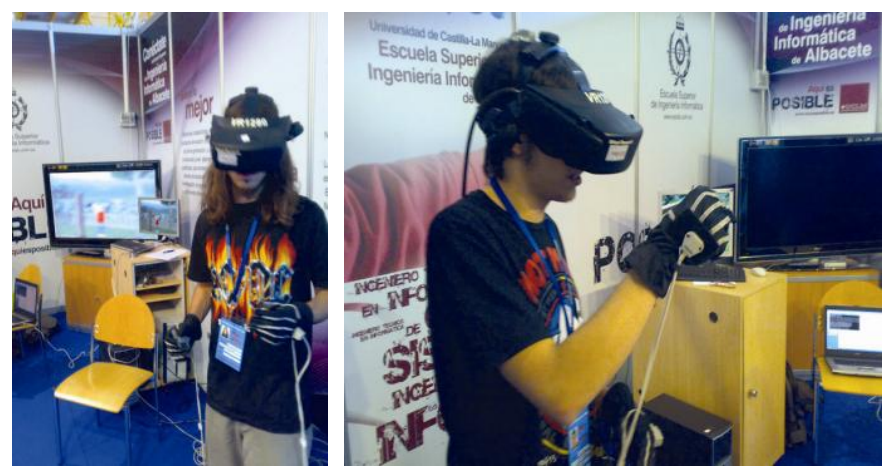

Fig. 6. A couple of users taking part in one of the trials 


\subsection{Conclusions of the experiment}

Couples using visual cues had an average of 0.77 beam falls per person each trial, and without them the average was 1 . So, an initial analysis of the results of this preliminary experiment shows that the hypothesis is right, but it is still necessary to analyze them in more detail.

Once the users finished the experiment, they were asked whether they found the visual cues useful or not. The results of the interview showed that $85 \%$ of the participants found them useful or very useful, while $15 \%$ did not pay much attention on them.

During the completion of this first experiment, several elements were identified that were considered important.

One is the position of the feedback cues within the CVE, since there are several alternatives, for example, showing them on the element that makes the action (partner's hand), on object that receives the action (beam) or on the display of the user. However, this element seems to be highly dependent on the application.

The possibility of changing the level of demand of the task was also considered. It can be done by changing the maximum length of the elastic band that connects the user's hands to the object, so that the shorter the elastic band the more attention users must pay to the task if they don't want the beam fall. This would show if the improvement in the performance due to the inclusion of specific cues for collaborative manipulation depends or not on the level of demand for the task at hand.

Surprisingly enough, it was observed that users did not frequently talk. Only $23 \%$ of the users that had visual aids available were talking while carrying out the task. Having in mind that this was a collaborative task, this percentage is too low. On the other hand, this percentage increased up to $40 \%$ when the users did not have visual cues. This may be related to the fact that, without visual cues, users had to overcome this shortcoming increasing the communication between them, something that was not as necessary having the visual aids available.

\section{CONCLUSIONS AND FUTURE WORK}

In this paper, it has been described a Collaborative Virtual Environment which has been used as a testbed to gain insight into how awareness and feedback can be used to improve collaborative manipulation in tasks that require closely-coupled collaboration. In the CVE developed, two users carried out one of the subtasks identified in [13] as a part of a general task of building a Gazebo.

Also, some recommendations have been given to CVE developers that have to include tasks that require close collaboration between users. These recommendations will help them to design the interaction in a more usable way.

The main objective of this study was to investigate whether the fact of introducing visual cues when carrying out a closely coupled collaborative task improve the user performance or the user experience. An initial analysis of the results of this preliminary evaluation showed that the awareness provided by the system improves the performance of the task.

However, a wider study is needed. Therefore, we are working on a new experiment based on the same task, so that more types of awareness can be analyzed. We are also planning to include audio and vibro-tactile cues.

Given that this new experiment will be considered an extension of the previous one, the same hypothesis will remain: the frequency of beam falls during the task will be greater in trials without sensory cues. In addition, taking into account what was observed during the first experiment, the level of demand of the task will be varied in order to check a second hypothesis: the awareness provided by the application becomes more necessary as the task becomes more demanding. This way, the maximum length of the elastic band will be varied.

\section{ACKNOWLEDGEMENT}

This work has been partially supported by the Ministerio de Educación y Ciencia of Spain (CICYT TIN2008-06596-C0201), and by the Junta de Comunidades de Castilla-La Mancha (PAI06- 0093).

\section{REFERENCES}

[1] J. Annet, Hierarchical Task Analysis. Lawrence Erlbaum Associates, 2003.

[2] J. Barrilleaux, 3D user interfaces with Java 3D. Manning Publications Co., 2001.

[3] C. Basdogan, C. Ho, M. Srinivasan, and M. Slater, "An experimental study on the role of touch in shared virtual environments," $A C M$ Transactions on Computer-Human Interaction 7, 4, 443-460, 2000.

[4] D. Bowman, J. Chen, C. Windgrave, J. Lucas, A. Ray, N. Polys, Q. Li, Y. Haciahmetoglu, J.-S. Kim, S. Kim, R. Boehringer, and T. Duval., "New directions in 3d user interfaces," in Proceedings of the 2005 IEEE Conference 2005 on Virtual Reality. IEEE Computer Society. 312-341, 2005.

[5] M. Fraser, T. Glover, I. Vaghi, S. Benford, C. Greenhalgh, J. Hindmarsh, and C. C.Heath, "Revealing the realities of collaborative virtual reality," Proceedings of the third international conference on Collaborative virtual environments (CVE '00). ACM Press. 29-37, 2000.

[6] S. Greenberg and D. Markwood, "Real time groupware as a distributed system: Concurrency control and its effect on the interface," in Proceedings of the ACM CSCW Conference on Computer Supported Cooperative Work. ACM Press, 1994.

[7] D. Margery, B. Arnaldi, and N. Plouzeau, "A general framework for cooperative manipulation in virtual environments," in Virtual Environments' 99: Proceedings of the Eurographics Workshop. Springer. 169-178., 1999.

[8] OpenSG, "http://opensg.vrsource.org/"

[9] O. Otto, D. Roberts, and R. Wolff, "A review on effective closelycoupled collaboration using immersive cve's," in Proceedings of the 2006 ACM international conference on Virtual reality continuum and its applications (VRCIA '06). ACM Press. 145-154., 2006.

[10] M. Pinho, D. Bowman, and C. Freitas, "Cooperative object manipulation in immersive virtual environments: Framework and techniques," in Proceedings of the ACM symposium on Virtual reality software and technology (VRST'02). ACM Press. 171-178., 2002.

[11] Raknet, "www.jenkinssoftware.com/"

[12] K. Riege, T. Holtkamper, G. Wesche, and B. Fr" ohlich, "The bent pick ray: An extended pointing technique for multi-user interaction," in 
Proceedings of $3 D$ User Interfaces (3DUI'06). IEEE Computer Society. 62-65., 2006.

[13] D. Roberts, R. Wolff, O. Otto, and A. Steed, "Constructing a gazebo: Supporting teamwork in tightly coupled, distributed task in virtual reality," Presence: Teleoperators and Virtual Environments 12, 6, 644-657, 2003.

[14] D. Roberts, O. Otto, and R. Wolff, "Pushmepullyou: The reality of interaction with shared objects in networked walk-in displays," in Proceedings of the ISCA 17th Internationsal Conference on Parallel and Distributed Computing Systems (PDCS'04). 470-477., 2004.

[15] D. Roberts, I. Heldal, O. Otto, and R. Wolff, "Factors influencing flow of object focussed collaboration in collaborative virtual environments," Virtual Reality Journal 10, 2, 119-133, 2006.

[16] R. Ruddle, J. Savage, and D. Jones, "Symmetric and asymmetric action integration during cooperative object manipulation in virtual environments," ACM Transactions on Computer-Human Interaction 9, 4, 285-308, 2002.

[17] K. Schmidt, "The problem with "awareness": Introductory remarks on "awareness in cscw"," Computer Supported Cooperative Work (CSCW).The Journal of Collaborative Computing 11, 3-4, 285-298, 2002.

[18] R. Schroeder, I. Heldal, and J. Tromp, "The usability of collaborative virtual environments and methods for the analysis of interaction," Presence: Teleoperators and Virtual Environments 15, 6, 655-667, 2006.

[19] VRJuggler, "http://www.vrjuggler.org/"

[20] R. Wolff, D. Roberts, A. Steed, and O. Otto, "A review of telecollaboration technologies with respect to closely coupled collaboration," International Journal of Computer Applications in Technology (IJCAT), Special Issue on: Collaborative Multimedia Applications in Technology 29, 1, 11-26, 2006.

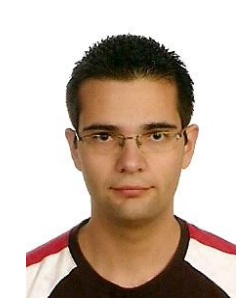

Arturo S. García received the MS degree in computer science from the University of Castilla-La Mancha in 2004. He is currently a $\mathrm{PhD}$ candidate at the LoUISE Lab of the University of Castilla-La Mancha. His research interests include CVE development and interaction improvement in VEs and CVEs.

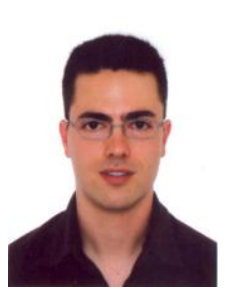

José P. Molina Dr. José Pascual MOLINA MASSÓ received the European Ph.D. in Computer Science from the University of Castilla-La Mancha (UCLM) in 2008. After receiving his Computer Engineering degree from the UCLM in summer 2000 , he spent a few months as a junior researcher at the IRI (Instituto de Rob'otica e Inform'atica Industrial, Barcelona) before coming back to UCLM by the end of that year. Since then, he has been an Assistant Professor of the Computing Systems Department, giving courses related to Computer Graphics and Virtual Reality. His research is focused in 3D User Interfaces, their design and evaluation, with several papers in relevant conferences and journals on Human-Computer Interaction.

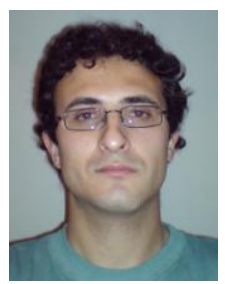

Diego Martínez is a $\mathrm{PhD}$ candidate at the LoUISE Lab of the University of Castilla- La Mancha, Spain. His research interests include VR systems and architectures, with special focus on CVE development. Contact him at diegomp1982@dsi.uclm.es.

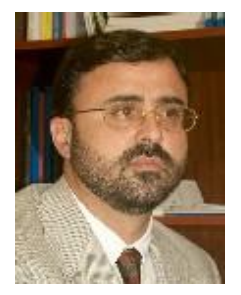

Pascual González Pascual González is MSc and $\mathrm{PhD}$ in Computer Science by the Polytechnic University of Madrid. Currently he is Associate Professor at the Department of Computer Systems of the University of Castilla-La Mancha and founder member and leader of the Laboratory of User Interaction and Software Engineering (LoUISE) at the Computer Science Research Institute of Albacete (University of Castilla-La Mancha). His researchinterests are, among others, human computer interaction, augmented and virtual reality, and software engineering applied to interactive systems. 